\title{
Functional model of water balance variability at the catchment scale: 2. Elasticity of fast and slow runoff components to precipitation change in the continental United States
}

\author{
C. J. Harman, ${ }^{1}$ P. A. Troch, ${ }^{2}$ and M. Sivapalan ${ }^{1,3,4}$ \\ Received 21 June 2010; revised 4 November 2010; accepted 1 December 2010; published 16 February 2011.
}

[1] Assessing the sensitivity of annual streamflow to precipitation is challenging due to the complexity of the processes that control the water balance. A low-dimensional model can be useful to interrogate data in regional assessments of a large number of catchments, and can provide insights into the broad similarities and differences between catchments' behaviors. This work assesses the sensitivity to precipitation of total annual streamflow, as well as its slow and fast flow components, within the framework of the Ponce and Shetty water balance model. This framework assumes that there are upper limits on the ability of a catchment to store and evaporate water, and there are minimum threshold amounts of precipitation and wetting (precipitation minus fast flow) needed to initiate fast and slow runoff. The flow elasticities (percent of change in flow per percent of change in annual precipitation) were estimated for 405 catchments in the Model-Parameter Estimation Experiment data set (including an assessment of parameter uncertainty). The elasticity of total discharge was 2.1 on average across the catchments, which is consistent with other studies. The fast component was higher $(\approx 2.4)$ and slow flow was lower $(\approx 2.0)$. Elasticities were highest and most variable in arid areas. The variations in elasticity between sites were shown to be primarily controlled by the two threshold parameters. The thresholds were a high proportion of mean annual precipitation in arid sites where only a small proportion of catchment wetting is released as slow flow. This provides some insight into previous observations that the sensitivity is correlated with climate.

Citation: Harman, C. J., P. A. Troch, and M. Sivapalan (2011), Functional model of water balance variability at the catchment scale: 2. Elasticity of fast and slow runoff components to precipitation change in the continental United States, Water Resour. Res., 47, W02523, doi:10.1029/2010WR009656.

\section{Introduction}

[2] Quantifying and predicting the effects of environmental change on catchment hydrology is important for socioeconomic reasons but is challenging due to the complexity of the processes that determine how water is partitioned in the landscape [Wagener et al., 2010]. Environmental changes do not occur in isolation from one another, and shifts in water balance will arise from both changes in the climatic drivers of catchment hydrology and from changes in the physical and ecological structure of landscapes, such as those associated with land use changes. To complicate matters, there are dynamic feedbacks between the climate, hydrologic response, and landscape biophysical processes over a wide variety of temporal and spatial scales. Evidence of such changes have

\footnotetext{
${ }^{1}$ Department of Civil and Environmental Engineering, University of Illinois at Urbana-Champaign, Urbana, Illinois, USA.

${ }^{2}$ Department of Hydrology and Water Resources, University of Arizona, Tucson, Arizona, USA.

${ }^{3}$ Department of Geography, University of Illinois at Urbana-Champaign, Urbana, Illinois, USA.

${ }^{4}$ Water Resources Section, Faculty of Civil Engineering and Geosciences, Delft University of Technology, Delft, Netherlands .
}

Copyright 2011 by the American Geophysical Union. 0043-1397/11/2010WR009656 already been observed (for instance) where long-term drought has led to the die-off of Piñon Pine in southwestern woodlands of North America [Breshears et al., 2005], altering the hydrologic behavior of these landscapes (M. Guardiola-Claramonte et al., Drought-induced tree die-off unexpectedly decreases stream flow in semi-arid basins, submitted to Journal of Hydrology, 2010).

[3] There is a great deal of previous work exploring the sensitivity of annual streamflow to its primary driver, precipitation, using a variety of methods [e.g., Budyko, 1974; Schaake, 1990; Milly, 1994; Arnell, 1999; Gellens and Roulin, 1998; Sankarasubramanian et al., 2001; Zhang et al., 2001; Woods, 2003; Chiew, 2006; Yang et al., 2007; Jothityangkoon and Sivapalan, 2009; Hurkmans et al., $2009,2010]$. These studies show that the sensitivity varies considerably between catchments due to a wide variety of factors. Those that have considered the partitioning at an annual scale, such as Budyko [1974] and Zhang et al. [2001], have only considered the partitioning of precipitation into streamflow and evapotranspiration, while in others the contributions of fast stormflow and slow base flow are determined by models (and parameters) that operate at faster time scales. Many of these studies use the elasticity of streamflows proposed by Schaake [1990] as a basis for analyzing the sensitivity to precipitation. 
[4] The annual water balance model of Ponce and Shetty [1995] described in the companion paper to this one (Sivapalan et al. [2011], which was itself based on the work of L'vovich [1979]) offers a basis for examining the partitioning into fast and slow flow components using functional parameters that have meaning at the annual time scale. These parameters are emergent properties, in the sense that they arise from the multitude of process interactions that control interannual variations in water balance. Sivapalan et al. [2011] have shown that this model provides robust predictions in catchments across the continental United States. That analysis revealed systematic spatial patterns in the annual water balance, and a space-time symmetry in which variations between catchments followed the same functional form as the variations in a single catchment between years.

[5] In this work we will use the Ponce and Shetty [1995] framework to quantify the sensitivity (in terms of the elasticity) of these fast and slow components to interannual variations in precipitation, and determine which of the functional parameters plays the most important role in determining the sensitivity. Moreover, the parameters' simplicity and parsimony allows for easier comparison between a large number of sites and the analysis of patterns of variation between sites. In this way we aim to observe how patterns in the spatial and (long-term) climatic controls on catchment function determine the sensitivity of the streamflow components to (short-term) climate variations (in this case precipitation).

\subsection{Space-for-Time and Time-for-Time Approaches to Understanding Water-Balance Sensitivity}

[6] In some works the variation in sensitivity seems to be largely associated with differences in aridity [Budyko, 1974; Schaake, 1990; Dooge, 1992]. Others have shown that snow cover and the phasing of water and energy inputs are important [Sankarasubramanian et al., 2001; Milly, 1994; Jothityangkoon and Sivapalan, 2009], as well as the capacity of catchments to store water and the "storminess" of the climate [Milly, 1994]. Woods [2003] developed coupled analytical models of the seasonal and annual water balance of catchments to show that six dimensionless parameters control hydrological partitioning: (1) climate dryness; (2) interception capacity relative to rainfall; (3) combined climate seasonality and root zone storage; (4) subsurface flow responsiveness; (5) saturated subsurface flow capacity, relative to mean annual rainfall rate; and (6) a geomorphological exponent controlling the expansion of saturated area fraction. Zhang et al. [2001] found that vegetation type showed a consistent effect on annual water balance, with higher evapotranspiration in forested catchments, and less in pasture (see Oudin et al. [2008] for an alternative perspective), though interannual variations were not examined. Yang et al. [2007] analyzed the spatial and temporal variability of annual water-energy balance in 108 nonhumid catchments in China and found that besides the annual climate conditions the regional pattern of annual water-energy balance was closely related to the relative infiltration capacity (hydraulic conductivity of the soil relative to the average rainfall intensity), relative soil water storage (maximum soil water storage relative to potential evapotranspiration), and the average slope of the catchment.
[7] The wide variety of climatic and landscape factors that control water balance greatly complicate the task of assessing regional patterns of sensitivity. The large amount of data required to characterize any particular catchment makes it difficult to use detailed process-based models to do such regional assessments of sensitivity, although they are certainly useful at the scale of individual catchments [Hurkmans et al., 2010, 2009]. The level of detail embedded in such models also obscures the fundamental similarities or differences in the ways variability propagates through catchments, controlled by the processes that partition, store, and release water (the emergent results of which Sivapalan [2005] called the "functions" of a catchment), and which control the way the sensitivity varies between catchments.

[8] One alternative to detailed modeling is to use data on the historical sensitivity to interannual variations in precipitation as a surrogate for the long-term effects [Risbey and Entekhabi, 1996]. This "time-for-time" substitution is based on the assumption that the changes in catchment response due to small climate shifts toward a wetter (or drier) climate are reasonably predicted by considering the behavior of catchments in the wetter (or drier) years it currently experiences. Typically the sensitivity is derived from a simple water balance model calibrated to each site [Němec and Schaake, 1982; Schaake, 1990; Chiew, 2006]. Such models have typically been applied for predicting changes in the partitioning of annual precipitation into annual evaporation and annual streamflow.

[9] The time-for-time approach ignores the effects that long-term shifts in climate might have on the catchment properties that control the water balance, such as the structure of the ecological community. Another alternative is to use data from an ensemble of catchments to look for patterns in the relationship between long-term average metrics of the climate and the partitioning of rainfall into runoff. Comparisons between catchments are made on the basis of dimensionless similarity indices that characterize the climate drivers and partitioning. The most well-known example of this approach is from Budyko [1974], who used longterm data from many catchments around the world to show that scaled evapotranspiration (scaled with respect to mean annual precipitation) depends primarily on the long-term humidity index (ratio of precipitation to potential evapotranspiration). This space-for-time substitution depends on the assumption that the trajectory of short-term (decades to century) responses of catchments to environmental change can be derived from the (dis)similarity between catchments that have been exposed to the projected climate and land cover conditions (e.g., more or less precipitation or conversion from grassland to forested landscapes) over long time periods (centuries to millennia) [Dooge, 1992; Dooge et al., 1999].

[10] The Ponce and Shetty [1995] model described by Sivapalan et al. [2011] has the advantages of the empirical models described above in that the predictions are based on the observed sensitivity. However, unlike the Budyko formulation or other typical annual water balance models, the L'vovich-Ponce and Shetty formulation enables the partitioning of runoff into quick and slow flow components. Thus, it provides more detailed insights into the patterns of climate and landscape controls on water balance. Perhaps most importantly, however, patterns in the functional 
parameters would allow the model to be used for both space-for-time and time-for-time substitution. These patterns would suggest that the effects of the long-term climate on the emergent properties of the hydrologic systems are encapsulated by the functional parameters. Taking advantage of these patterns of the emergent properties, we could propose that short-term variations in climate are characterized by the interannual variability parametrized in the Ponce and Shetty model, while long term variations are described by shifts in the parameters themselves according to the observed between-site patterns.

\subsection{Outline of This Work}

[11] The paper is structured as follows. We begin by deriving an elasticity metric of sensitivity within the framework of the Ponce and Shetty model. We then apply these metrics to the Model-Parameter Estimation Experiment (MOPEX) data set of United States catchments, which has also been used to investigate controls on the water balance in the continental United States by Sivapalan et al. [2011], S. Zanardo (Climatic and landscape controls on inter-annual variability of catchment water balance and vegetation wateruse: A probabilistic approach, submitted to Water Resources Research, 2011), and H. Voepel et al. (Quantifying the role of climate and landscape characteristics on hydrologic partitioning and vegetation response, submitted to Water Resources Research, 2011). In order to determine the robustness of this elasticity parameter (given the scatter in the fit of the model to the annual water balance data) we then use a Markov chain Monte Carlo algorithm to determine uncertainty bounds on the sensitivity metrics for each catchment in the database. This analysis is important to establish the credibility of the predicted sensitivities.

[12] We then investigate which of the functional parameters most strongly determine the sensitivity, and examine the spatial and climatic variations in these parameters. We will examine relationships of these parameters with the humidity index (the ratio of annual precipitation and potential evapotranspiration $P\left(E_{P}\right)$ and the Horton index. The Horton index is a metric of the annual water balance equal to the fraction of the wetting $W$ that goes toward vaporization $V$ (i.e., $H=V / W$ ) and was the subject of a detailed study by Troch et al. [2009]. The results are then compared to previously published analyses of sensitivity.

[13] It is important to note from the start that this framework does not account for many of the other sources of interannual variability, such as variations in temperature and energy inputs more generally, and the timing of rainfall within the year. Temperature in particular may exhibit systematic changes over time that affect hydrologic partitioning. For instance, an increase in temperature could increase the vaporization potential and/or increase the slow flow runoff potential. These changes could be accounted for by allowing the parameters (and particularly the vaporization potential in the case of variations in energy inputs) to be functionally dependent on other climatic inputs. However, this is beyond the scope of the current work.

\section{Methods}

[14] The Ponce and Shetty model was originally described by Ponce and Shetty [1995], and further elabo- rated in the companion paper to this one [Sivapalan et al., 2011]. Readers are referred to those papers for details of the model. In brief though, the model assumes that at an annual time scale all incoming precipitation $P$ is partitioned into a fast component of runoff $S$ and a "wetting" component $W$. The wetting component is then further partitioned into a slow component of runoff $U$ and evapotranspiration, which is called vaporization $V$ for reasons of consistency with the previous works. No carry-over storage between years is accounted for. Each of the two partitioning steps is determined by two parameters: (1) an upper limit, known as the wetting potential $W_{p}$ and vaporization potential $V_{P}$, and (2) a threshold for runoff generation $\lambda_{s} W_{P}$ and $\lambda_{u} V_{P}$ (we will treat these products as single parameters, which preserves the nomenclature of Ponce and Shetty [1995] but keeps the definition of the threshold independent of the potentials). These parameters all have units of millimeters (volume per catchment area $\left[\mathrm{L}^{3} / \mathrm{L}^{2}\right]$ ). Total annual discharge (per unit catchment area) is the sum of the components $Q=S+U$. Note that in practice, the fast and slow components are identified using hourly or daily data and a base flow separation algorithm, and the totals of each flow component are aggregated at the annual scale. Troch et al. [2009] suggest that the choice of algorithm is not a significant determinant of the water balance at the annual scale.

\subsection{Sensitivity in the Ponce and Shetty Framework}

[15] Following Schaake [1990] and Dooge [1992] we will use the economic metric of "elasticity" to quantify the sensitivity of catchment water balance to input variability. This metric is generically defined as the percent change in one variable given a unit percent change in another. The use of elasticity is preferable to a simple rate of change, as it elegantly captures the impact of a given change on the normal state of a system. If a decrease in annual precipitation of $10 \mathrm{~mm}$ leads to a decrease in annual streamflow of $30 \mathrm{~mm}$, this might be a negligible change in the humid tropics, but would be highly significant in a semiarid catchment. This difference would be reflected in different values of the elasticity metric in each catchment. Considering the change in streamflow per change in precipitation, we can use the approximation of the derivative to write

$$
\Delta Q=\Delta P \frac{\partial Q}{\partial P}
$$

and similar expressions with $U$ and $S$ substituted for $Q$, where $P$ is the annual precipitation. Rearranging this relationship we can write

$$
\begin{aligned}
& \frac{\Delta S}{S}=\rho_{S} \frac{\Delta P}{P}, \\
& \frac{\Delta U}{U}=\rho_{U} \frac{\Delta P}{P}, \\
& \frac{\Delta Q}{Q}=\rho_{Q} \frac{\Delta P}{P},
\end{aligned}
$$

where the elasticity metrics $\rho$ that give the percent of change in the water balance component per percent of change in precipitation are defined as 


$$
\begin{gathered}
\rho_{S}=\frac{\partial S}{\partial P} / \frac{S}{P}, \\
\rho_{U}=\frac{\partial U}{\partial P} / \frac{U}{P}, \\
\rho_{Q}=\frac{\partial Q}{\partial P} / \frac{Q}{P} .
\end{gathered}
$$

[16] Note that since $Q=S+U$ and $\partial Q=\partial S+\partial U$, we can write

$$
\frac{\partial Q}{\partial P}=\frac{\partial S}{\partial P}+\frac{\partial U}{\partial P}
$$

[17] Thus it is possible to obtain the elasticity of the total discharge from its components $S$ and $U$ and their elasticities

$$
\rho_{Q}=\frac{S}{S+U} \rho_{S}+\frac{U}{S+U} \rho_{U}
$$

[18] Expressions for the $\rho$ parameters can be found by substituting the Ponce and Shetty model in the definitions and taking the partial derivatives. Note that it remains necessary to specify the precipitation values at which the sensitivity is required. If we specify that we are interested in the sensitivity around the mean $\bar{P}$, the modeled partition values are given by

$$
\begin{aligned}
\bar{S} & =\frac{\left(\bar{P}-\lambda_{s} W_{P}\right)^{2}}{W_{P}-2 \lambda_{s} W_{P}+\bar{P}}, \\
\bar{W} & =\frac{W_{P} \bar{P}-\lambda_{s} W_{P}^{2}}{W_{P}-2 \lambda_{s} W_{P}+\bar{P}}, \\
\bar{U} & =\frac{\left(\bar{W}-\lambda_{u} V_{P}\right)^{2}}{V_{P}-2 \lambda_{u} V_{P}+\bar{W}},
\end{aligned}
$$

where $\bar{P}$ is the mean precipitation. Values of the required partial derivatives with respect to $P$ are then given by

$$
\begin{aligned}
& \left.\frac{\partial S}{\partial P}\right|_{\bar{P}}=\frac{2\left(\bar{P}-\lambda_{s} W_{p}\right)}{W_{p}-2 \lambda_{s} W_{p}+\bar{P}}-\frac{\left(\bar{P}-\lambda_{s} W_{p}\right)^{2}}{\left(W_{p}-2 \lambda_{s} W_{p}+\bar{P}\right)^{2}}, \\
& \left.\frac{\partial W}{\partial P}\right|_{\bar{P}}=\frac{W_{p}}{W_{p}-2 \lambda_{s} W_{p}+\bar{P}}-\frac{W_{p} \bar{P}-\lambda_{s} W_{p}^{2}}{\left(W_{p}-2 \lambda_{s} W_{p}+\bar{P}\right)^{2}}, \\
& \left.\frac{\partial U}{\partial P}\right|_{\bar{P}}=\frac{2 \frac{\partial W}{\partial P}\left(\bar{W}-\lambda_{u} V_{p}\right)}{V_{p}-2 \lambda_{u} V_{p}+\bar{W}}-\frac{\frac{\partial W}{\partial P}\left(\bar{W}-\lambda_{u} V_{p}\right)^{2}}{\left(V_{p}-2 \lambda_{u} V_{p}+\bar{W}\right)^{2}} .
\end{aligned}
$$

[19] With these equations we can calculate the sensitivity parameter $\rho$ evaluated around the mean.

\subsection{MOPEX Data Set: Parameter Estimation and Uncertainty}

[20] The Ponce and Shetty model was applied to 405 catchments from the MOPEX data set [Schaake et al.,
2006; Duan et al., 2006] used by Sivapalan et al. [2011]. This data set is a standardized collection of up to 50 years of precipitation and flow data from catchments across the United States (available at www.nws.noaa.gov/oh/mopex). The subset of catchments selected from the database differ slightly from those of the companion paper, in that only catchments with at least 25 years of flow data available were used, and no catchments were excluded on the basis of parameter identifiability. They range in size from 80 to more than $10,000 \mathrm{~km}^{2}$, are distributed across the United States, but are mostly east of the Rocky Mountains, and are subject to a range of climates, with mean annual precipitation ranging from 436 to $2380 \mathrm{~mm}$ and humidity index (precipitation $P$ divided by potential evapotranspiration $E_{P}$ ) ranging from 0.25 to 2.5 . Quick and slow flow components were separated in the streamflow data using a one-parameter low-pass filter (see Sivapalan et al. [2011] for full discussion), and totals were obtained over the water year (1 October to 30 September). Troch et al. [2009] demonstrated that the estimation of annual water balance metrics, such as the Horton index, was not highly sensitive to the method of base flow separation.

[21] While previous work has estimated the Ponce and Shetty parameters for the MOPEX data set, the uncertainty in these parameters has not been quantified. Uncertainty in the functional parameters arises due to the scatter in the relationship between annual precipitation and the streamflow components. Because of this scatter, different combinations of parameters may produce an equally acceptable fit to the data. This uncertainty propagates to the estimates of the elasticity, as these are calculated from the functional parameters. Given that we intend to make predictions of sensitivity of streamflow to climate change, in this work it is important to determine this uncertainty.

[22] Parameters were estimated in a Bayesian framework with uninformative priors, which provide a rigorous framework for assessing uncertainty with a minimum of assumptions. From the initial fit we determined that the errors in the prediction of $S$ and $U$ were close to normally distributed for the MOPEX data set. For the purposes of the parameter estimation, it was assumed that the observations of $S$ and $U$ were drawn from a normal distribution with pdf $N\left[x \mid\left(\mu, \sigma^{2}\right)\right]$ in which the mean is given by the Ponce and Shetty model predictions $S=S\left(P, W_{P}, \lambda_{s} W_{P}\right)$ and $U=U\left(P, V_{P}, \lambda_{u} V_{P}\right)$, and the variances are the unknown variables $\sigma_{S}^{2}$ and $\sigma_{U}^{2}$. Note that errors in the prediction of $S$ and $U$ are perfectly inversely correlated with errors in $W$ and $V$, and so these latter errors can be ignored. The likelihood $L(\Omega \mid \theta)$ of the observations $\Omega=\left\{S_{1}, \ldots, S_{n}, U_{1}, \ldots, U_{n}\right\}$ given a model $\theta=$ $\left\{W_{P}, \lambda_{s} W_{P}, V_{P}, \lambda_{u} V_{P}, \sigma_{S}^{2}, \sigma_{U}^{2}\right\}$ with input precipitation time series $P_{1}, \ldots, P_{n}$ is given by the product

$$
\begin{aligned}
L(\Omega \mid \theta)= & \left(\prod_{i=1}^{n} N\left(S_{i} \mid S\left(P_{i}, W_{P}, \lambda_{S} W_{P}\right), \sigma_{S}^{2}\right)\right) \\
& \left(\prod_{i=1}^{n} N\left(U_{i} \mid U\left(P_{i}, V_{P}, \lambda_{u} V_{P}\right), \sigma_{U}^{2}\right)\right) .
\end{aligned}
$$

[23] From Bayes theorem, the posterior likelihood of the model given the data $L(\theta \mid \Omega)$ is given by 


$$
L(\theta \mid \Omega)=\frac{L(\Omega \mid \theta) L(\theta)}{L(\Omega)}
$$

where $L(\theta)$ is the prior distribution of the parameters, and $L(\Omega)$ is the probability of the observations. As we have no reason to constrain the prior distribution, we will use a uniform prior distribution, and therefore set $L(\theta)=1$. While $L(\Omega)$ is required to obtain a properly scaled posterior distribution, it is not necessary to evaluate it when using the methods described below.

[24] The posterior distribution was constructed by sampling the parameter space $\theta$ using the Metropolis algorithm [Kuczera and Parent, 1998; Metropolis et al., 1953]. This algorithm is a Markov chain Monte Carlo technique for efficiently sampling in the vicinity of maximum likelihood, while also providing good coverage of areas of lower likelihood. For each site the algorithm was run to generate a chain of 2500 samples. Convergence was determined as the point in the chain at which the mean over blocks of 250 consecutive samples changed by less than half the standard deviation of the entire chain. Samples prior to convergence of the chain were discarded. This procedure was then repeated to generate a total of 10,000 samples of the (unscaled) posterior likelihood at each site. Finally, by substituting the parameters of each sample into equation (2), likelihoods could be associated with values of the elasticity at each site.

[25] The best estimate of each parameter and elasticity was chosen as the maximum likelihood parameter set. The lower and upper uncertainty bounds were defined as the values for which the sum of the likelihoods of lower values was just greater than 5 and $95 \%$ of the total. In the remainder of this paper the error is reported in absolute terms as half the range between the upper and lower uncertainty bounds, or in relative terms as a percentage of the maximum likelihood value.

\section{Results}

\subsection{Sensitivity of Streamflow Components to Precipitation}

[26] Results of the parameter estimation and elasticity are summarized in Table 1. The elasticities ranged from 1.02 to 8.38 for $Q, 1.70$ to 6.08 for $S$, and 0.75 to 10.96 for $U$. Average values of the elasticities across all sites was highest for the quick flow $S$ at 2.38, lowest for the slow flow $U$ at 1.94 , and intermediate at 2.08 for $Q$. However, the variability between sites (measured by the standard deviation across sites) was smallest for the quick flow
(0.57), and highest for slow flow (0.88), with variability in the elasticity of total flow between these $(0.70)$. The elasticities of the fast and slow flow components, however, were loosely correlated $\left(R^{2}=0.62\right)$.

[27] The relationship between the elasticities and the humidity index $\left(P / E_{P}\right)$ is shown in Figure 1. The elasticities are generally larger in dry regions, but also show the greatest variability among sites. The scatter seems to be enclosed by two envelopes: an upper bound with a roughly hyperbolic shape that declines with humidity, and a lower bound that is invariant with climate. These bounds appear to converge for humidity index greater than 2, although there are only a handful of sites in the data set that are that humid. At those sites the elasticities are highly consistent between sites: $\rho_{S} \approx 1.83, \rho_{U} \approx 1.13$, and $\rho_{Q} \approx 1.30$.

[28] These climatic patterns create distinct spatial patterns, as shown in Figure 2. All three elasticities are highest in a band running north-south along the continental United States. The sensitivities in the band between $89^{\circ} \mathrm{W}$ and $99^{\circ} \mathrm{W}$ are higher than the national average by 0.60 for $Q$, 0.51 for $S$, and 0.66 for $U$.

\subsection{Robustness of Functional Parameters and Sensitivity Results}

[29] Median absolute errors in the functional parameters were $907 \mathrm{~mm}$ (or $19 \%$ of the best estimates of parameter values) for $W_{p}, 364 \mathrm{~mm}$ (24\%) for $V_{P}, 84 \mathrm{~mm} \mathrm{(65 \% )} \mathrm{for}$ $\lambda_{s} W_{P}$, and $100 \mathrm{~mm}(70 \%)$ for $\lambda_{u} V_{P}$. The percent errors for the thresholds are skewed because of the high relative error where the best estimate of the threshold is small, since an error of a given size is a large percentage of a "best" value close to zero. Best estimates of parameters coincided closely with those in Sivapalan [2005], which were fitted using a simple nonlinear least-squares regression.

[30] The median uncertainty in the elasticities were $8.0 \%$ $\left(\rho_{Q}\right), 10.3 \%\left(\rho_{S}\right)$, and $11.8 \%\left(\rho_{U}\right)$, which is small compared to the range of variation between sites. This gives us confidence that we can make valid comparisons between sites. Absolute errors in the potentials were generally correlated with the best estimate, as were the errors in the threshold when they were less than approximately $50 \mathrm{~mm}$ (results not shown).

\subsection{Controls of the Functional Parameters on Sensitivity}

[31] The runoff generation thresholds $\lambda_{s} W_{P}$ and $\lambda_{u} V_{P}$ had a much greater control over the elasticity than the wetting and vaporization potentials. We can illustrate the

Table 1. Overview of the Estimated Parameters for the Ponce and Shetty Model and Derived Elasticities for the MOPEX Dataset ${ }^{\mathrm{a}}$

\begin{tabular}{|c|c|c|c|c|c|c|}
\hline & Minimum & Mean & Maximum & SD & $\begin{array}{l}\text { Median Abs. } \\
\text { Error }\end{array}$ & $\begin{array}{c}\text { Median Rel. } \\
\text { Error (\%) }\end{array}$ \\
\hline$W_{P}$ & 1746 & 7635 & 123,200 & 10,386 & 906 & 18.9 \\
\hline$V_{P}$ & 75 & 3246 & 117,081 & 10,317 & 364 & 24.1 \\
\hline$\lambda_{U} V_{P}$ & 7.15 & 210 & 617 & 152 & 100 & 69.6 \\
\hline$\rho_{Q}$ & 1.02 & 2.08 & 8.38 & 0.70 & 0.15 & 7.98 \\
\hline$\rho_{S}$ & 1.70 & 2.38 & 6.08 & 0.57 & 0.24 & 10.28 \\
\hline$\rho_{U}$ & 0.75 & 1.94 & 10.96 & 0.88 & 0.20 & 11.79 \\
\hline
\end{tabular}

aMinimum, mean, maximum, and SD of the best estimates are shown, indicating the distribution across all the sites of the (individual sites' best estimate of) parameters and elasticities. The median absolute and relative error refer to the median level (between sites) of the uncertainty in sites' pa rameter and elasticity estimates. 

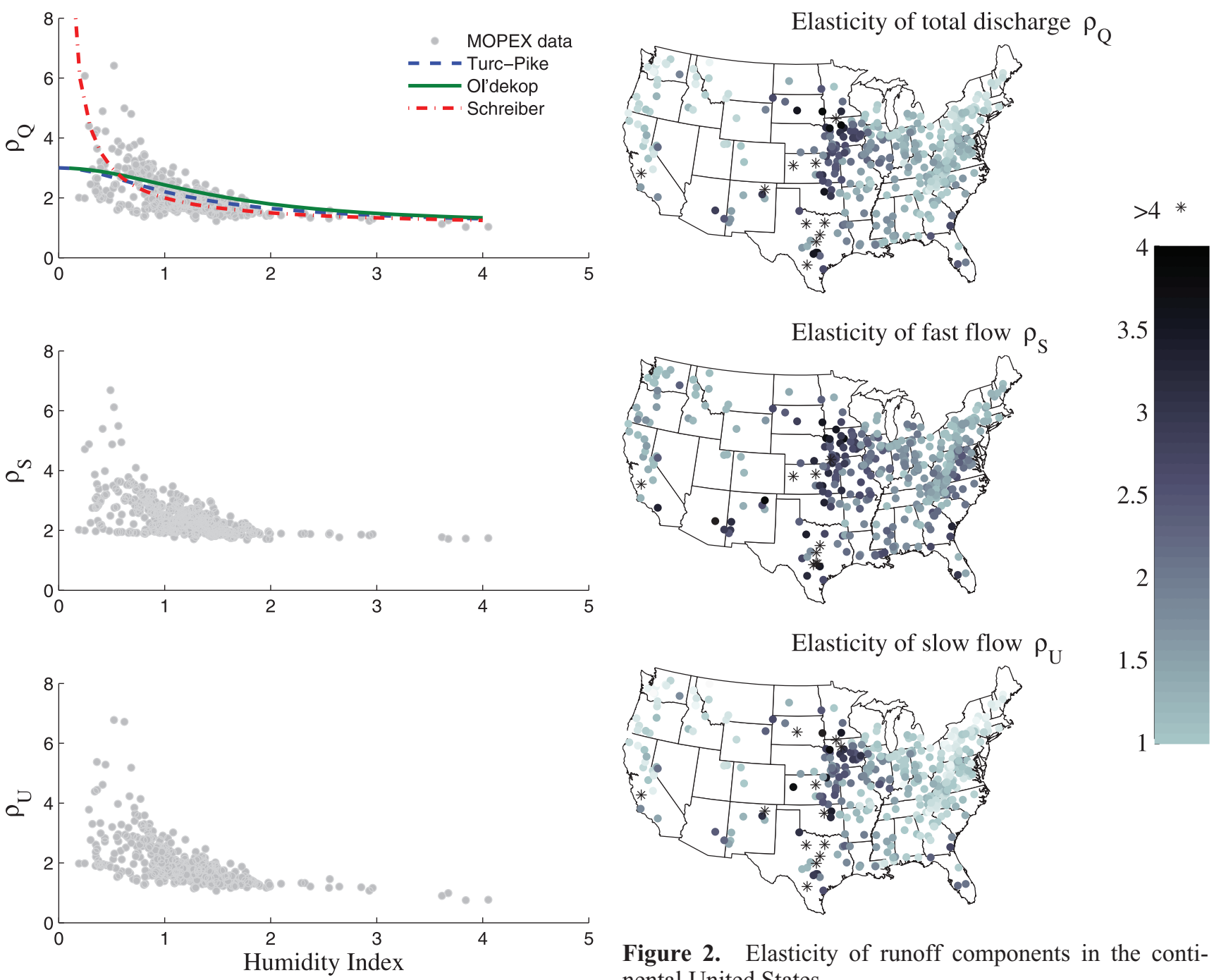

Figure 1. Relationships between elasticity and the humidity index $P / E_{P}$. Elasticity is higher for total flow and both components in the arid sites, and converges to a common value in humid sites. Three relationships cited by Dooge [1992] are plotted for comparison.

degree to which the differences in elasticity between sites are controlled by the functional parameters by plotting each elasticity against each parameter, as shown in Figure 3. Although both $W_{p}$ and $\lambda_{S} W_{P}$ control $\rho_{S}$, and all four parameters control $\rho_{U}$ and $\rho_{Q}$, the thresholds are by far the most important controls. In particular, the fast flow threshold almost uniquely determines the fast flow elasticity. Both exert very strong controls over the other elasticities.

[32] The data suggest that the climate and long-term water balance exert some control on the thresholds. Plotting the threshold functional parameters, normalized by mean annual precipitation, against the mean annual Horton index (Figure 4), shows that in arid catchments (where Horton indices $\sim 1$ ) the thresholds are a large proportion of annual precipitation, while in humid catchments (particularly where $\mathrm{H}$ is less than about 0.4 ), the thresholds are close to 0 . Because the impact of the threshold on the water balance depends on its magnitude relative to the annual precipita-

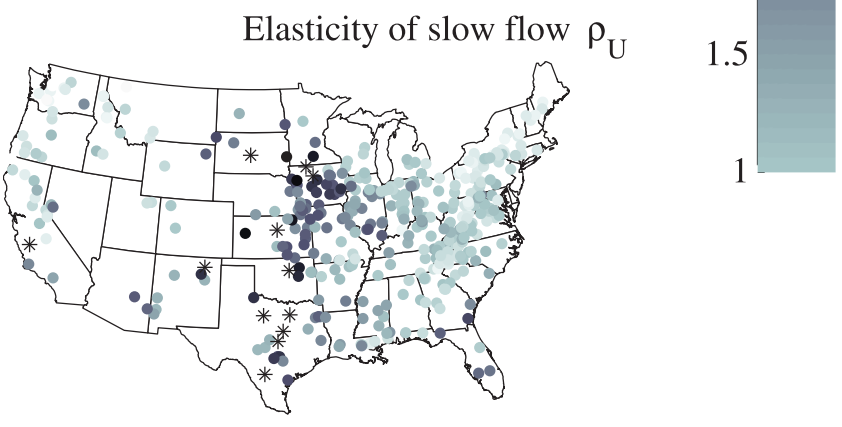

Figure 2. Elasticity of runoff components in the continental United States.

tion and wetting, this result implies that the thresholds exert a large control on the runoff in arid areas, and a smaller control in humid areas. To some extent the increasing relationship between the normalized threshold and $\mathrm{H}$ observed in Figure 4 is simply the result of the lower precipitation in arid areas used to do the normalization, rather than a systematic variation in the thresholds themselves, and so this result must be interpreted with care.

$$
\frac{\lambda_{s} W_{P}}{\bar{P}}=\Lambda_{S} \approx\left\{\begin{array}{ll}
1.46 \times(\bar{H}-0.584) & \bar{H}>0.584 \\
0 & \bar{H} \leq 0.584
\end{array}\left(R^{2}=0.46\right)\right.
$$

$\frac{\lambda_{u} V_{P}}{\bar{P}}=\Lambda_{U} \approx\left\{\begin{array}{ll}2.30 \times(\bar{H}-0.622) & \bar{H}>0.622 \\ 0 & \bar{H} \leq 0.622\end{array}\left(R^{2}=0.24\right)\right.$

\section{Discussion}

\subsection{Functional Controls on Climate Sensitivity}

[33] Why are the thresholds such an important control on the elasticity, rather than the upper potentials? The 


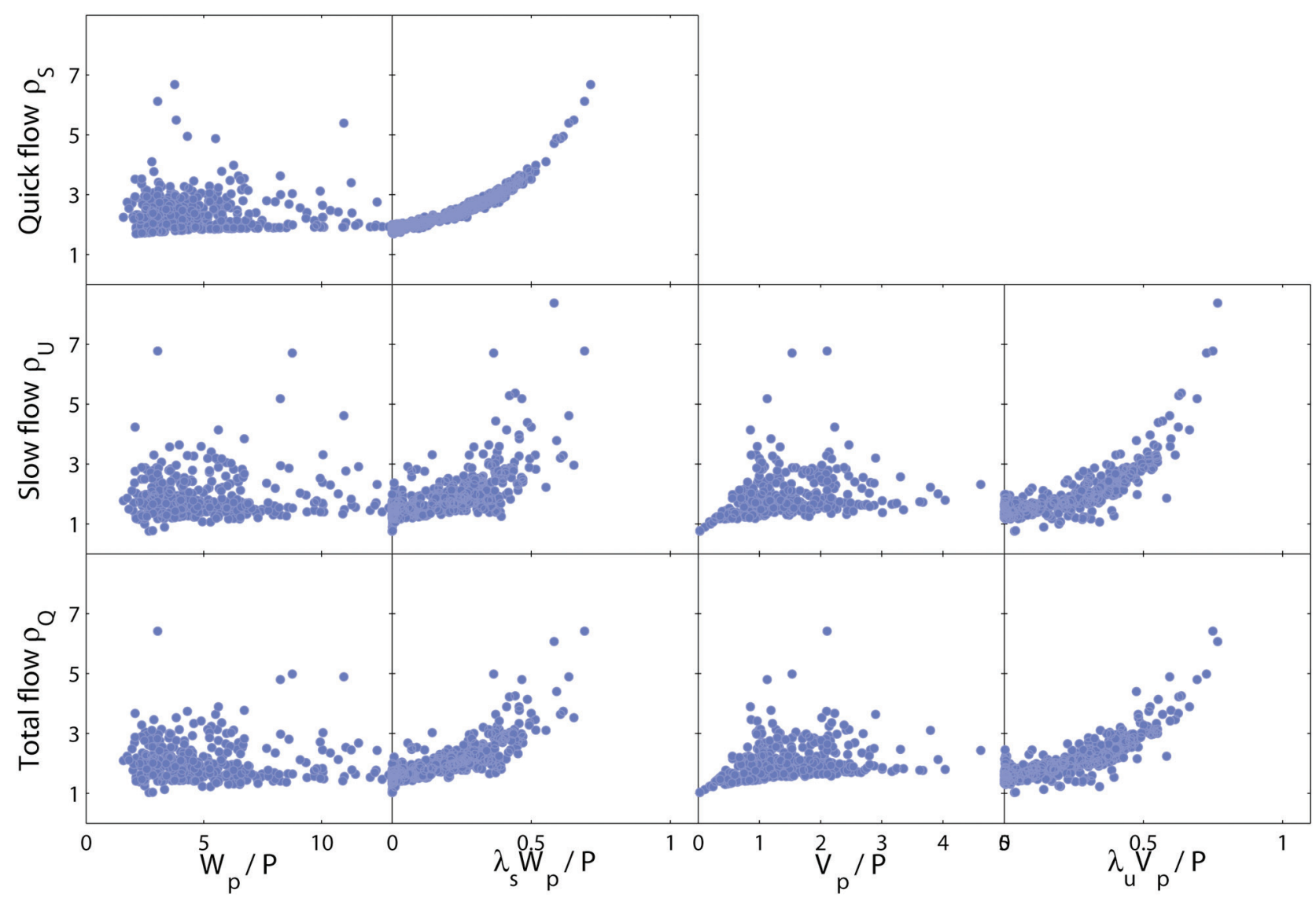

Figure 3. Relationship between the predicted elasticity and the functional parameters (normalized by $P)$. Differences between sites' sensitivity is mostly explained by differences in their threshold parameters $\lambda_{s} W_{P}$ and $\lambda_{u} V_{P}$. The upper potential $W_{P}$ and $V_{P}$ have less relative control.

importance of the thresholds arises largely because of the control they exert on the baseline amount of runoff generated and to which any increase is compared. This is illustrated in Figure 5, which shows the relationship between quick flow and precipitation at two sites. The two sites have very similar mean $P$ and $W_{p}$, but different $\lambda_{s} W_{P}$. Both have a mean annual rainfall around $980 \mathrm{~mm}$, and have similar wetting potentials around $4000 \mathrm{~mm}$. The Genesee River site at Wellsville, New York, has a threshold of $\lambda_{s} W_{P}=100 \mathrm{~mm}$, while the Embarras River at Sainte
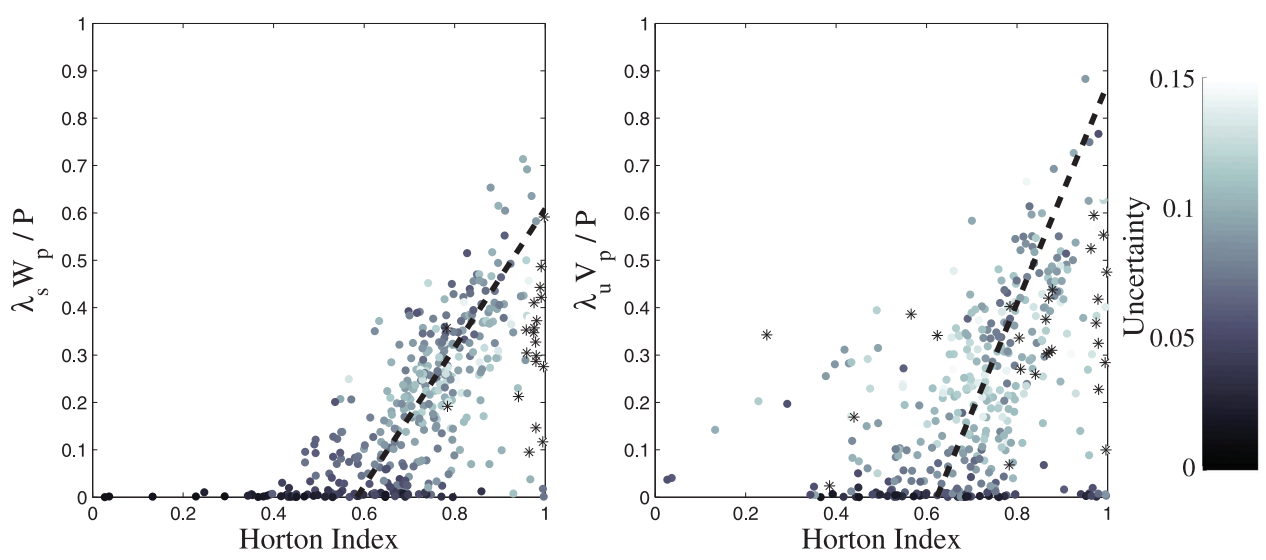

Figure 4. The thresholds for runoff production (normalized by annual precipitation) are directly correlated with the Horton index. Absolute uncertainty in the threshold parameters is shown by the tone (uncertainty greater than 0.15 is given by an asterisk). 


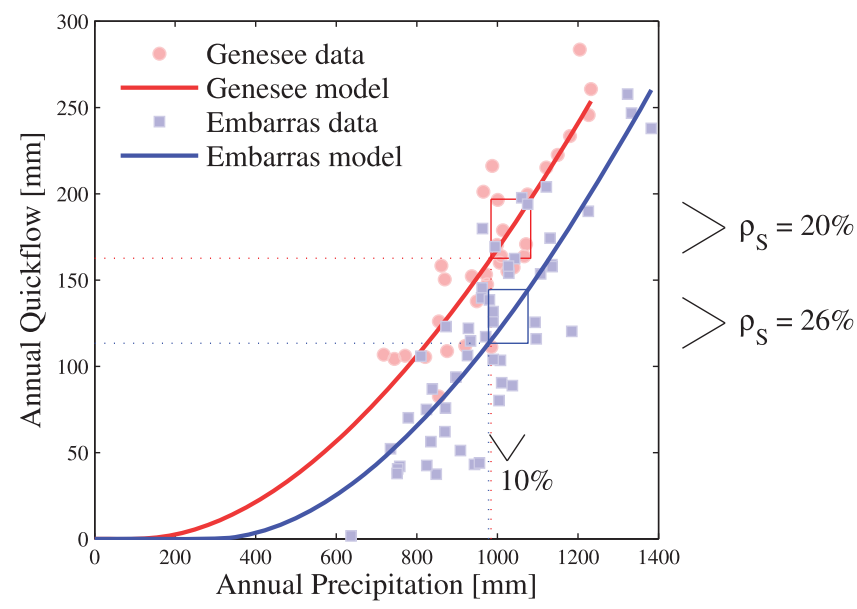

Figure 5. Quick flow as a function of precipitation for two very similar sites that differ mainly in their quick flow thresholds. See the text for a discussion. nate the thresholds from the expressions for the elasticities (equation (2)), and then rearranging the Ponce and Shetty model predictions of the Horton index at the mean annual precipitation (given by Sivapalan et al. [2011]) to eliminate the vaporization potential $V_{P}$. With this procedure we obtain

$$
\begin{gathered}
\frac{S}{P}=\frac{\left(1-\Lambda_{S}\right)^{2}}{W_{P} / \bar{P}+1-2 \Lambda_{S}}, \\
\frac{U}{P}=\frac{(1-H)\left(W_{P} / \bar{P}-\Lambda_{S}^{2}\right)}{W_{P} / \bar{P}+1-2 \Lambda_{S}}, \\
\rho_{S}=\frac{2 W_{P} / \bar{P}+1-3 \Lambda_{S}}{\left(1-\Lambda_{S}\right)\left(W_{P} / \bar{P}+1-2 \Lambda_{S}\right)},
\end{gathered}
$$

$$
\rho_{U}=\frac{\left(W_{P} / \bar{P}-\Lambda_{S}\right)^{2}\left[(H+1) W_{P} / \bar{P}+4 \Lambda_{S} \Lambda_{U}-2 \Lambda_{U}\left(W_{P} / \bar{P}+1\right)-(H+1) \Lambda_{S}^{2}\right]}{\left(W_{P} / \bar{P}+1-2 \Lambda_{S}\right)\left[\Lambda_{S}^{2}-2 \Lambda_{S} \Lambda_{U}+\left(\Lambda_{U}-1\right) W_{P} / \bar{P}+\Lambda_{U}\right]^{2}}
$$

Marie, Illinois, has $\lambda_{s} W_{P}=288 \mathrm{~mm}$. Consequently, the mean annual quick flow at the former site is larger: 165 $\mathrm{mm}$ as opposed to $117 \mathrm{~mm}$. Under a $10 \%$ increase in precipitation (i.e., $98 \mathrm{~mm}$ ) both of these increase by about the same amount (34 and $31 \mathrm{~mm}$ ). However, because of the difference in the baseline amount of quick flow, the latter is a larger percentage increase. The value of $\rho_{S}$ at the former is therefore lower at 2.0, while at the latter it is 2.6. Despite the scatter in the relationships shown in Figure 4, these sites follow the general pattern: the Genesee River site (with the lower threshold) is wetter than the Embarras River site, with a higher humidity index (1.4 versus 1.0$)$ and lower Horton index ( 0.64 versus 0.81$)$.

[34] It could be argued that it is unsurprising to find a relationship between the Horton index and the slow flow threshold, since this parameter was calibrated against the same data that was used to determine the mean Horton index. However, the threshold is determined by the interannual variability, and not just the mean of the data. Furthermore, there is no reason to expect a relationship between the Horton index and the fast flow threshold because the Horton index is determined by the partitioning that occurs after the fast flow threshold has had its influence; yet this is the strongest of the relationships.

[35] The relationship between the thresholds and the Horton index offers an opportunity to simplify the analysis of the links between the long-term water balance partitioning and the interannual variability. It is possible to express the sensitivities in terms of only two variables, the mean annual Horton index and a normalized wetting potential $W_{P} / \bar{P}$. This is achieved by using equations (18) and (19) to elimi- where for convenience we have used the shorthand $\Lambda_{S}$ and $\Lambda_{U}$ for the estimated normalized thresholds calculated using equations (18) and (19). These expressions (plus equation (9)) can be used to predict the elasticity of runoff over the short term in terms of a long-term average water balance property, the Horton index, and $W_{P} / \bar{P}$. Figure 6 compares the elasticities obtained from the MOPEX database with this function for fixed values of $W_{P} / \bar{P}$. The values of $W_{P} / \bar{P}$ used are representative of median (4.5) 10th percentile (2.7) and 90th percentile (10.1) in the MOPEX data. First, this figure demonstrates that the elasticities of all the components of runoff are functions of the mean Horton index. This is an interesting result, as it links the interannual variability of runoff to the long-term average hydrologic functionality, as captured by the Horton index.

[36] Second, it further demonstrates that the thresholds are the primary control on the runoff elasticity, particularly in dry climates. The gray lines illustrate the variation in the elasticity predicted by the Ponce and Shetty model if the thresholds are fixed at zero. In those cases, the elasticity is bounded at a maximum of 2 , in reality it can be much higher. The wetting potential $W_{P} / \bar{P}$ is a second-order control on the elasticity, at best. Much of the scatter in the relationship is therefore due to other processes affecting interannual variability that is not accounted for in this model.

\subsection{Comparison With Other Predictions of Climate Sensitivity}

[37] The results are directly comparable to other time-fortime type analyses of precipitation sensitivity that appear in the literature. Sankarasubramanian et al. [2001] derived a 

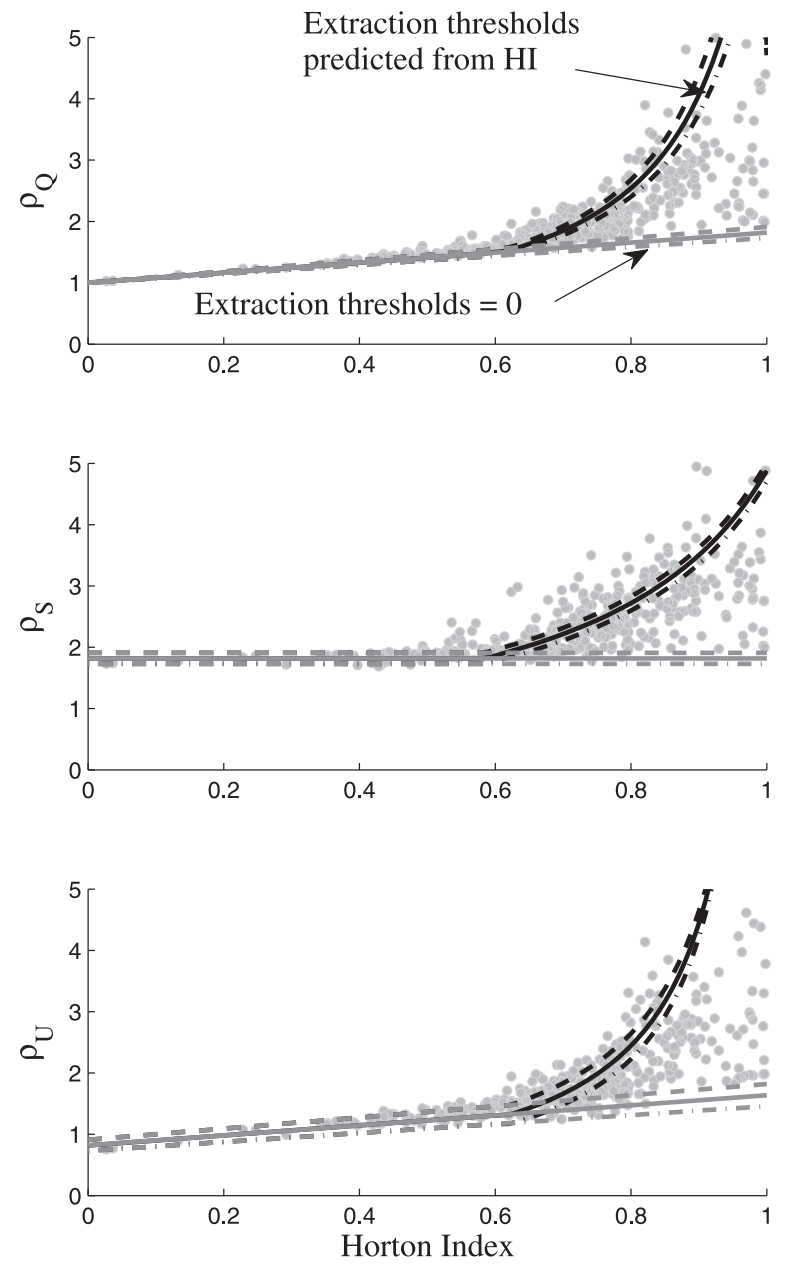

Figure 6. Relationship between the elasticity and the Horton index. Curves are given by equation (20). Black lines use thresholds predicted by regression, while the grey lines show the sensitivity when the thresholds are set to 0 . The dashed, solid, and dash-dot lines represent different values of $W_{P} / \bar{P}$. Values used are representative of median (4.5) 10th percentile (2.7) and 90th percentile (10.1) in the MOPEX data.

map of streamflow sensitivities to changes in precipitation for the United States, based on at least 20 years of observations from 1291 catchments. They used a nonparametric estimator, compared it with model-based estimates of streamflow sensitivities in three catchments using Monte Carlo experiments, and concluded that the nonparametric estimator is as or more robust, less biased, and independent of model structure and parameter estimation procedures. Figure 7 compares the elasticities of total flow in the MOPEX data set calculated using the Ponce and Shetty model and this nonparametric metric. The result shows that the methods are comparable, though the nonparametric method tends to predict lower values for the lower and upper ends of the distribution. The lower end values have greater uncertainty in the Ponce and Shetty model.

[38] Based on the mapped results Sankarasubramanian et al. [2001] observed that snow dominated catchments tend to have the lowest streamflow sensitivities, as do catchments with very high humidity indices. Arid and

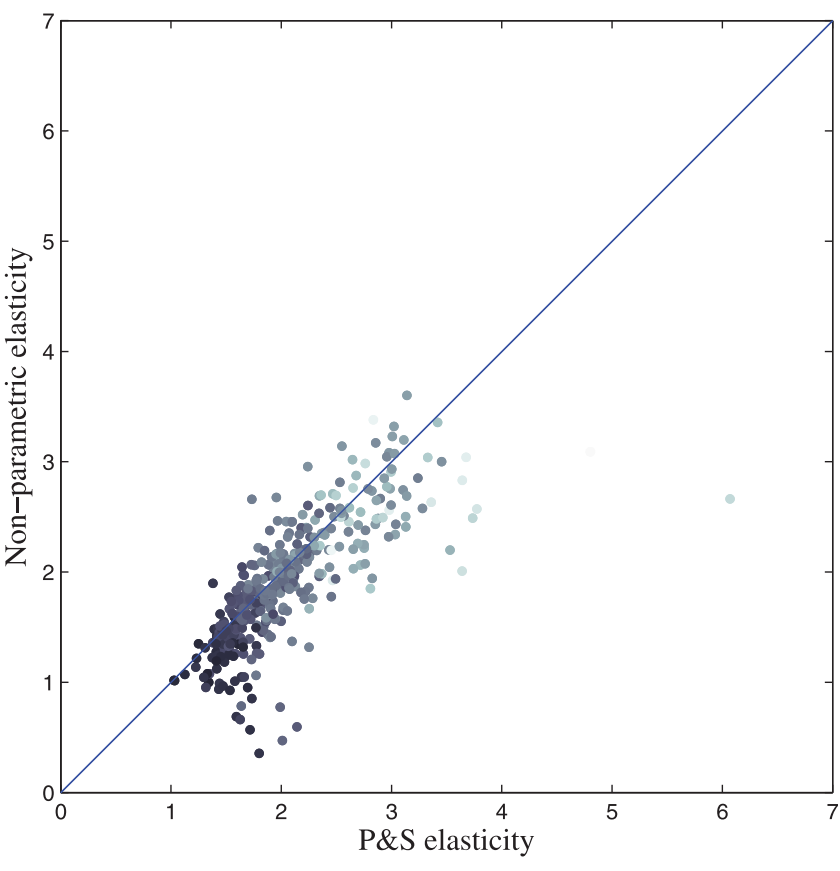

Figure 7. Comparison of estimated elasticity from the Ponce and Shetty model with the nonparametric approach of Sankarasubramanian et al. [2001]. The nonparametric approach predicts lower elasticities at the upper and lower end of the distribution. Tone represents the relative uncertainty (light tone is higher relative uncertainty).

semiarid catchments tend to have the highest streamflow sensitivities, and regions where moisture and energy availability are out of phase have very high spatial variation of sensitivities. They compared their results with the results from Dooge [1992] who used the Budyko hypothesis to derive streamflow sensitivities, and concluded that the Budyko hypothesis is only valid in regions with high humidity indices, but that in semiarid catchments the nonparametric estimator yields considerable scatter around the theoretical values derived by Dooge [1992].

[39] In Figure 1 we have plotted the sensitivities of total streamflow (Figure 1a), quick flow (Figure 1b), and slow flow (Figure 1c) of all 405 catchments as functions of the mean humidity index. (Figure 1 can be compared to Figure 8 from Sankarasubramanian et al. [2001]). As from Sankarasubramanian et al. [2001], the streamflow sensitivity seems to converge to a value of 1 for very humid catchments. This result is consistent with Dooge [1992] who derived analytical expressions of streamflow sensitivity to precipitation changes based on three empirical relationships (Ol'dekop, Turc-Pike, and Schreiber) that comply with Budyko's hypothesis. (These equations are empirical approximations of the Budyko relationship. The reader is referred to Dooge [1992] for details.) However, for very dry catchments our results indicate that streamflow sensitivity becomes very large and beyond the limit value of 3 as predicted by Ol'dekop and Turc-Pike parametrizations. The main difference between our results and those of Sankarasubramanian et al. [2001] is the predicted streamflow sensitivities for semiarid regions. Our estimates never fall below the horizontal asymptote of 1 , whereas the results 
reported in Sankarasubramanian et al. [2001] vary dramatically from very low (near zero) to high (4.5) sensitivities.

[40] Sankarasubramanian et al. [2001] found that semiarid catchments with very low streamflow sensitivity are located in Missouri and Pacific Northwest water resource regions [Seaber et al., 1987]. The western part of the Missouri water resource region is poorly covered by the MOPEX database, but MOPEX catchments located in the Pacific Northwest used in this study never exhibit streamflow sensitivities less than 1 . The reason for this discrepancy is unclear. Part of it is due to the different method used, which introduces systematic differences at high and low values. It may also be due to the fact that different sets of catchments were used. The large variation of streamflow sensitivities can also be related to intra-annual variations in moisture and energy availability, as pointed out by Sankarasubramanian et al. [2001]. Finally, it is possible that a negative covariance between annual precipitation and temperature that may exist in some of these semiarid catchments (hot and dry versus cold and wet) is not taken into account in the Ponce and Shetty model. More research is needed to shed light on the possible causes of these differences, and to account for them.

[41] In Dooge's work, Schreiber's method predicts infinite streamflow sensitivity as the humidity index approaches 0 , and this seems to fit the general trend of our results well (Figure 1). Moreover, the general trend of all flow component sensitivities with humidity index is very similar. It is interesting to note that quick flow sensitivity seems to converge to a value slightly less than 2 for very humid catchments, while slow flow sensitivity converges to a value slightly more than 1 . This suggests that for these humid regions, even though total streamflow changes proportionally to changes in precipitation, quick flow will experience the greatest change.

\section{Conclusions}

[42] While many previous studies have examined streamflow elasticity to changes in precipitation [Schaake, 1990; Němec and Schaake, 1982; Dooge, 1992; Risbey and Entekhabi, 1996; Dooge et al., 1999; Sankarasubramanian et al., 2001], the perspective and insights offered in this work are unique. The Ponce and Shetty approach parametrizes the way catchments partition, store, and release water; that is their function, abstracted to a high level. This approach has allowed us to make the several new observations, which warrant reiteration.

[43] The Ponce and Shetty model divides discharge into fast and slow components, allowing the sensitivity of these to be assessed independently. It was observed that in United States catchments in the MOPEX database, estimates of the elasticity of total discharge were found to be around 2.1 on average; the fast component of streamflow tended to have a higher elasticity, at around 2.4 on average, indicating that this component was more sensitive to interannual variability in precipitation; the elasticity of slow flow was lower at around 1.9. The observation of different elasticities for each flow component is significant for understanding how the structure of the water balance may change under a changing climate.

[44] The functional model allowed us to look for systematic controls on the observed elasticity in terms of the thresholds and the potentials that encapsulate the catchment function. We conclude that the threshold values exert the strongest control. In the case of quick flow the threshold for generating quick flow almost entirely controls the elasticity. This was found to be because these parameters are the primary determinants of the underlying amount of flow that any change is being compared to.

[45] Moreover, there is a relationship between these threshold values and the long-term hydrologic partitioning through the Horton index $H=V / W$. The thresholds were a high proportion of mean annual precipitation in arid sites (that is, with a low-humidity index), and at sites where $H$ is large. $V$ is mainly water use by vegetation and $W$ is related to water holding capacity of the catchment. Both $V$ and $W$ are determined by the catchment characteristics such as vegetation type and soils [see also $\mathrm{H}$. Voepel et al., submitted manuscript, 2011; Zanardo, submitted manuscript, 2011]. Specifically, $H$ combines ecosystem and soil response to climate drivers $\left(P\right.$ and $\left.E_{p}\right)$ and as such captures how catchments have evolved (in terms of ecosystem dynamics and storage capacity) under prevailing climate [see also Sivapalan et al.. 2011; Troch et al., 2009].

[46] We were able to derive analytical expressions that relate the elasticities to long-term mean $\mathrm{H}$, and those expressions capture very well the general relationship between sensitivity and $\mathrm{H}$. These relations can form the basis for a space-for-time substitution to understand how catchments may respond to climate change. We thus effectively combined time-for-time with space-for-time approaches to hydrologic change analysis. The derived elasticities predict catchment response to short-term (decades) change in $P$, while the relationships between the sensitivities and Horton index (i.e., humidity index) suggest how catchment response will change at larger time scales (centuries to millennia) when the system has had time to adapt to such changes in climate and have evolved toward an equilibrium state.

[47] Deriving a more robust method for linking spacefor-time and time-for-time, and deeper insights into the nature of the functional controls on catchment water balance, will depend on being able to better link the thresholds (and the upper potentials) to the landscape properties that control them. The thresholds are the result of many process interactions that may be unique to a particular place. Understanding how they emerge serves as a basis for making comparisons between catchments even where these processes differ. A full understanding of the physical controls on these emergent processes is an important avenue for future work. The functional parameters offer a basis for framing questions about controls on catchment behavior that can be answered using more detailed physically based hydrologic models.

[48] The functional parameters used here arise from the interactions of a raft of processes and factors operating at subannual times scales, such as the seasonality of the climate, the timing of storm inputs, and vegetation phenology. These processes are themselves subject to other sources of interannual variability, and these contribute to the scatter in the observed partitioning, and uncertainty in the appropriate functional parameters. An important example is the role of interannual variations in temperature on the amount of precipitation that falls as snow, and the rate and timing of snowmelt. It is possible to imagine an extension to the 
model that incorporates an additional partitioning of snowfall (separate from rainfall) into sublimation versus snowmelt. However, such an extension makes little sense without considering interannual variations in temperature, and must be left for future work.

[49] The functional parameters also provide a basis for examining the kinds of changes in landscape biophysical properties over time that might occur either due to exogenous forces such as global climate change, or endogenous changes such as land use and land cover change. The difference in sensitivity between the fast and slow flow components identified here are especially interesting given that changes in the fast and slow flow components of the hydrograph are often associated with some distinct classes of water management issues (e.g., water quality issues are often associated with high flows, while in-stream habitat availability during dry periods are associated with low flows). Hypotheses can be quite easily generated in this framework. For example, many of the processes of urbanization in a catchment may lead to a reduction in the fast runoff threshold and wetting potential as impervious areas replace infiltrating surfaces. Replacement of deep-rooted perennials with shallow-rooted annual crops may lead to a reduction in the vaporization potential. Extraction of groundwater from superficial aquifers may lead to increases in the slow flow threshold, as more rainfall is required to generate significant subsurface flow toward streams. All these hypotheses can be easily tested given a sufficiently long pretreatment and posttreatment record. Alternatively, the framework described here can be used to interpret the effects of changes in parameters or inputs on detailed models of water balance, and so generate more generalizable insights.

[50] More work is also required to investigate further controls on the annual water balance, and how these will shift in the future. In areas where snow is an important control on the water balance, shifts in snow versus rainfall and the timing of rainfall are known to be very important [Leung et al., 1999; Nijssen et al., 2001]. It is also particularly important to understand how intra-annual variability (seasonality, phase of precipitation cycles relative to cycles of energy availability, etc.) further affects the sensitivity.

[51] Acknowledgments. Work on this paper commenced during the Summer Institute organized at the University of British Columbia (UBC) during June-July 2009 as part of the NSF-funded Hydrologic Synthesis project, "Water Cycle Dynamics in a Changing Environment: Advancing Hydrologic Science through Synthesis" (NSF grant EAR-0636043, M. Sivapalan, PI). We acknowledge the support and advice of numerous participants at the Summer Institute (students and faculty mentors). Partial support was provided by NSF grants EAR09-11,205 (PI: Sivapalan) and EAR06-35,752 (PI: Wagener). Special thanks due to Mateij Durcik for help with the background data preparation and analysis of the MOPEX data set. Thanks also due to Marwan Hassan and the Department of Geography of UBC for hosting the Summer Institute and for providing outstanding facilities, without which this work would not have been possible. The authors would also like to thank Andrew Neal, Paul Brooks, and Sally Thompson, whose feedback on a draft manuscript greatly improved the final paper.

\section{References}

Arnell, N. W. (1999), A simple water balance model for the simulation of streamflow over a large geographic domain, J. Hydrol., 217(3-4), 314335, doi:10.1016/S0022-1694(99)00023-2.

Breshears, D. D., et al. (2005), Regional vegetation die-off in response to global-change-type drought, Proc. Nat. Acad. Sci. USA, 102(42), 15,144-15,148, doi:10.1073/pnas.0505734102.
Budyko, M. I. (1974), Climate and Life, 508 pp., Elsevier, New York.

Chiew, F. H. S. (2006), Estimation of rainfall elasticity of streamflow in Australia, Hydrol. Sci. J., 51(4), 613-625.

Dooge, J. (1992), Sensitivity of runoff to climate change: A Hortonian approach, Bull. Am. Meteorol. Soc., 73, 2013-2013.

Dooge, J., M. Bruen, and B. Parmentier (1999), A simple model for estimating the sensitivity of runoff to long-term changes in precipitation without a change in vegetation, Adv. Water Resour., 23, 153-163.

Duan, Q., et al. (2006), Model Parameter Estimation Experiment (MOPEX): An overview of science strategy and major results from the second and third workshops, J. Hydrol., 320(1-2), 3-17.

Gellens, D., and E. Roulin (1998), Streamflow response of Belgian catchments to IPCC climate change scenarios, J. Hydrol., 210(1-4), 242-258.

Hurkmans, R. T. W. L., W. Terink, R. Uijlenhoet, E. J. Moors, P. A. Troch, and P. H. Verburg (2009), Effects of land use changes on streamflow generation in the Rhine basin, Water Resour. Res., 45(6), 1-15, W06405, doi:10.1029/2008WR007574.

Hurkmans, R., W. Terink, R. Uijlenhoet, P. Torfs, D. Jacob, and P. A. Troch (2010), Changes in streamflow dynamics in the Rhine Basin under three high-resolution regional climate scenarios, J. Clim., 23(3), 679, doi:10.1175/2009JCLI3066.1.

Jothityangkoon, C., and M. Sivapalan (2009). Framework for exploration of climatic and landscape controls on catchment water balance, with emphasis on inter-annual variability, J. Hydrol., 371, 154168, doi:10.1016/ j.jhydrol.2009.03.030.

Kuczera, G., and E. Parent (1998), Monte Carlo assessment of parameter uncertainty in conceptual catchment models: The Metropolis algorithm, J. Hydrol., 211(1-4), 69-85, doi:10.1016/S0022-1694(98) 00198-X.

Leung, L. R., and M. S. Wigmosta (1999), Potential climate change impacts on mountain watersheds in the Pacific Northwest, J. Am. Water Resour. Assoc., 35(6), 1463-1471, doi:10.1111/j.1752-1688.1999.tb04230.x.

L'vovich, M. (1979), World Water Resources and Their Future, 415 p., AGU, Washington, D. C.

Metropolis, N., A. Rosenbluth, M. Rosenbluth, and A. Teller (1953), Equations of state calculations by fast computing machines, J. Chem. Phys, 21(6), 1087-1092.

Milly, P. C. D. (1994), Climate, soil water storage and the average annual water balance, Water Resour. Res., 30(7), 2143-2156.

Němec, J., and J. Schaake (1982), Sensitivity of water resource systems to climate variation, Hydrol. Sci. J., 27(3), 327-343.

Nijssen, B., G. M. O’Donnell, A. F. Hamlet, and D. P. Lettenmaier (2001), Hydrologic sensitivity of global rivers to climate change, Clim. Change $50(1), 143-175$.

Oudin, L., V. Andréassian, J. Lerat, and C. Michel (2008), Has land cover a significant impact on mean annual streamflow? An international assessment using 1508 catchments, J. Hydrol., 357(3-4), 303-316.

Ponce, V., and A. Shetty (1995), A conceptual model of catchment water balance: 1. Formulation and calibration, J. Hydrol., 173(1-4), 27-40, doi:10.1016/0022-1694(95)02739-C.

Risbey, J., and D. Entekhabi (1996), Observed Sacramento Basin streamflow response to precipitation and temperature changes and its relevance to climate impact studies, J. Hydrol., 184, 209-223.

Sankarasubramanian, A., R. M. Vogel, and J. F. Limbrunner (2001), Climate elasticity of streamflow in the United States, Water Resour. Res., 37(6), 1771-1781, doi:10.1029/2000WR900330.

Schaake, J. C. (1990), From climate to flow, in Climate Change and U.S. Water Resources, edited by P. E. Waggoner, chap. 8, pp. 177-206, Wiley, New York.

Schaake, J., S. Cong, and Q. Duan (2006), The US MOPEX data set, IAHSAISH Publ., 307, 9-28.

Seaber, P. R., F. P. Kapinos, and G. L. Knapp (1987), Hydrologic unit maps, Water Suppl. Pap. 2294, 61 pp., U.S. Geol. Surv., Denver, Colo.

Sivapalan, M. (2005), Pattern, process and function: Elements of a unified theory of hydrology at the catchment scale, in Encyclopedia of Hydrological Sciences, edited by M. G Anderson, pp. 193-219, Wiley, New York.

Sivapalan, M., M. A. Yaeger, C. J. Harman, X. Xu, and P. A. Troch (2011), A functional model of water balance similarity at the catchment scale: Space-time symmetry of hydrologic variability, Water Resour. Res., doi:10.1029/2010WR009568, in press.

Troch, P. A., G. F. Martinez, V. R. N. Pauwels, M. Durcik, M. Sivapalan, C. Harman, P. D. Brooks, H. Gupta, and T. Huxman (2009), Climate and vegetation water use efficiency at catchment scales, Hydrol. Process., 23(16), 2409-2414, doi:10.1002/hyp.7358. 
Wagener, T., et al. (2010), The future of hydrology: An evolving science for a changing world, Water Resour. Res., 46(5), 1-10, W05301, doi:10.1029/2009WR008906.

Woods, R. (2003), The relative roles of climate, soil, vegetation and topography in determining seasonal and long-term catchment dynamics, $A d v$ Water Resour., 26, 295-309.

Yang, D., F. Sun, Z. Liu, Z. Cong, G. Ni, and Z. Lei (2007), Analyzing spatial and temporal variability of annual water-energy balance in nonhumid regions of China using the Budyko hypothesis, Water Resour. Res., 43, W04426, doi:10.1029/2006WR005224.
Zhang, L., W. R. Dawes, and G. R. Walker (2001), Response of mean annual evapotranspiration to vegetation changes at catchment scale, Water Resour. Res., 37, 701-708, doi:10.1029/2000WR900325.

C. J. Harman, M. Sivapalan, and P. A. Troch, Hydrosystems Building MC 250, Department of Civil and Environmental Engineering, University of Illinois at Urbana-Champaign, $205 \mathrm{~N}$ Matthews Ave., Urbana, IL 61801, USA. (charman2@illinois.edu) 